\title{
Achievement Emotions and Peer Acceptance Get Together in Game Design at School Margherita Brondino $o^{\mathrm{a}}$, Gabriella Dodero ${ }^{\mathrm{b}}$, Rosella Gennari ${ }^{\mathrm{b}}$, A- lessandra Melonio ${ }^{\mathrm{b}}$, Daniela Raccanello ${ }^{\mathrm{a}}$, Santina Torello ${ }^{\mathrm{b}}$ \\ margherita.brondino@univr.it, gabriella.dodero@unibz.it, gennari@inf.unibz.it, alessandra.melonio@unibz.it, da- niela.raccanello@univr.it, santina.torello@unibz.it \\ ${ }^{a}$ Department of Philosophy, Education and Psychology, University of Verona, Lungadige Porta Vittoria 17, 37129 Verona, Italy \\ ${ }^{\mathrm{b}}$ Faculty of Computer Science, Free University of Bozen-Bolzano, Piazza Domenicani 3, 39100 Bolzano, Italy
}

KEYWORD

Game design; Participatory design; Gamification; Cooperation; Children; Sociometric status; Achievement emotions

\author{
ABSTRACT
}

\section{Introduction}

\subsection{Rationale}

A game design study with and for children was conducted at school, in Spring 2014. Using a participatory design approach, children were asked to create their own game ideas, in the form of documents, and to realize their game ideas in paper format as low-fidelity prototypes. Children were organized in groups of 3-5 members using cooperative learning. Game design tasks and classrooms were gamified, and ad-hoc gamified material was created for designing, following gamification principles. The blend of gamification and cooperative learning in a participatory game design experience aimed at eliciting children's positive emotions and social inclusion. 
Social inclusion can be conceptualized in terms of peer acceptance, one of the components of social competence as defined by Waters and Sroufe (1983). The other components are social engagement, social behavioral and psychological profiles, and peer acceptance. On the whole, social competence plays a key role for individuals' endeavors to organize behavioral, cognitive, and affective resources to achieve relevant goals within a group. Similarly, achievement emotions, which are focused on achievement activities or outcomes, are assumed to be central for outcomes related to performance in learning environments by theoretical approaches such as the control-value theory (Pekrun, 2006; Pekrun \& Perry, 2014). In addition, control and value beliefs-as constructs highly relevant in participatory game design processes-would be critical in determining such emotions. However, so far research has paid scarce attention to the relationships between social competence and achievement emotions, and specifically on how peer acceptance can predict achievement emotions and how, in turn, it can be influenced in a game design context.

\subsection{Novelty and Outline}

Nowadays, design studies at school generally aim at including all children and engaging them emotionally in the experience as well, e.g., (Garzotto, 2008). The study reported in this paper has such aims as well. The majority of stud-ies, however, are of a qualitative nature. To the best of our knowledge, (Brondino et al., 2015) is the first research study that takes care of operationalizing and measuring both social inclusion and children's emotions in a design ac-tivity with groups of children, as in quantitative research. This paper is an extended version of (Brondino et al. 2015; submitted a), in which discrete achievement emotions were considered. This paper, for the first time, explores and operationalizes inclusion in different social contexts. It has thus novel analyses for inclusion and its relations with achievement emotions, filling a gap in both the education and game design literatures alike.

The game design method used with children is outlined in the first part of this paper. The second part reports the study ran in primary schools: it explains how inclusion and emotions were measured, and results concerning their links. The paper concludes assessing the study results and implications for future design experiences with and for children.

\section{The Design Method}

\subsection{Introduction}

Gamified Co-design with Cooperative Learning (GaCoCo) is rooted in participatory design, and has developed techniques for prototyping games with children at school. Conceived in (Dodero et al., 2014a), it was incrementally refined across studies for allowing children to design games at school. An example of a transition from a study to another is reported in (Dodero et al., 2014b). Its ideas were then applied also in different domains, e.g., for gamifying dissemination activities for classes of children in a university context (Del Fatto et al., 2014).

In GaCoCo, children work on early game design in groups of 3-5. Teachers illustrate the work organization and material to be used, according to the daily design protocol for children. More generally, teachers are in classroom with researchers for managing and explaining the protocol to children. GaCoCo researchers are usually two per class. One is the expert designer, who follows each group for providing rapid feedback during the daily design session, and for conducting a formative evaluation of each group product at the end of the session, the results of which are made available in the follow-up session for fixing design.

In order to manage social relations and organize work of groups of children, GaCoCo relies on a proven instructional methodology: cooperative learning. Strategies for organizing the work of groups, rules and roles for children are all important contributions that $\mathrm{GaCoCo}$ acquires from cooperative learning and adapts to the end of game design, making them tangible via gamified material. GaCoCo uses gamifica- tion in order to create material making tangible not 
only cooperative learning but, more generally, the design protocol for children itself. In such a manner, GaCoCo aims at sustaining children's positive engagement in design, in particular when this is split across different design days, across different weeks. The material for designing is created by the game design expert with experts of child development and tested, with the aim of being engaging and usable.

The remainder of this section delves into the main contributions of cooperative learning and gamification in GaCoCo: strategies, rules and roles from cooperative learning; game design principles and gamified material from gamification.

\subsection{Cooperative Learning Contributions}

Cooperative learning is an instructional methodology, based on constructivism, for managing group work. How this can be promoted varies according to the chosen cooperative learning models. Hereby we refer to the Complex Instruction model of cooperative learning by Cohen (Cohen, 1998).

The model structures class work for small heterogeneous groups, so as to promote the visibility of all, leveraging on the different skills of members. Heterogeneity becomes a growth opportunity for all. In particular, heterogeneity elicits group creativity: learners' early design solutions are triggered by diverse perspectives; these allow group members to build various design alternatives (Paulus \& Nijstad, 2003). Cooperative learning strategies for heterogeneous groups, rules and roles are all important contributions that GaCoCo adapts to the end of design.

\subsubsection{Strategies}

There are a number of strategies for organizing the work of heterogeneous groups in cooperative learning and that GaCoCo picked up (Kagan \& Kagan, 1992). Hereby we give examples of strategies relevant for this paper, for groups consisting of 3-5 members. In think-pair-share, group members work in pairs as follows: first the class teacher or domain expert poses a question; then each learner thinks individually about a response and hence shares ideas with their partner in an attempt to reach a solution to the problem. Instead, in a three-step-interview, children in each group take part in an interview or a discussion divided into three steps, each of c. 2-3 minutes. The domain expert or teacher introduces the topic and each group member chooses another member as partner. In the first step, partners interview each other by asking clarification questions. Secondly, partners are swapped. In the final third step, groups are recreated and discuss the topic.

\subsubsection{Roles}

In $\mathrm{GaCoCo}$, roles for design are not fixed but rotate among members, so as to train different skills. The group ambassador is an example of a role for children spanning across GaCoCo design activities. Ambassadors ask for clarifications; more generally, they are responsible of exchanging information with the teacher and design expert.

The secretary is another role recurring in GaCoCo activities: secretaries take notes on project development and group decisions with dedicated material. However it is the material manager who is always in charge of collecting the right material from the expert when foreseen by the GaCoCo protocol.

\subsubsection{Rules}

Besides strategies and roles, and in support of them, cooperative learning considers a set of rules necessary for group work, and for including all. Rules are concerned with social skills, such as reciprocal listening and respect of different views. Examples of cooperative learning rules that GaCoCo employs across design activities are: rule for turns: tak- 
ing turns in voicing opinions; rule for reconciliation: reconciling different views, e.g., concerning game design documents or prototypes.

As outlined below, GaCoCo has gamified material for making tangible strategies, roles and rules for relating to others.

\subsection{Gamification Contributions}

Game design, from mechanics to aesthetics, is behind the broad area of gamification (Hunicke et al., 2004). In its most common acceptation, gamification means: properly using game-based elements, such as story lines and progression bars, for a non-game goal and in a non-game context in order to positively engage people regarded as players, e.g., see (Kapp, 2012).

Kapp (2012) lists diverse motivation theories for explaining why gamification can trigger positive emotional engagement. In general, gamification should nourish a sense of social relatedness, as well as competence and progression, control and autonomy, and have a powerful feedback system. Let us see how that is designed for in GaCoCo.

\subsubsection{Relatedness}

Relatedness needs are important components of games, and are supported in GaCoCo via cooperative learning, as explained above. Gamified material can help in making tangible cooperative learning for connecting with others. Examples of such material are signaling disks and scepters for sharing with others, illustrated in Figure 1 and used in the 2014 study. A signaling disk is used for sharing and voting ideas in groups, reconciling different views (rule for reconciliation of cooperative learning): children can draw smileys or write their feedback, positive or not, on their signaling disks in relation to the voting task. A scepter is used for sharing and organizing the turn to speak among group members (rule for turns of cooperative learning).

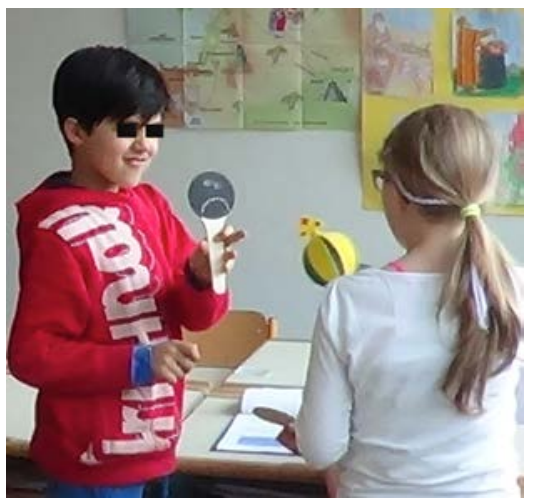

Figure 1. Signalling discks (left, black) and scepters (right, yellow) for relating to others

\subsubsection{Competence and Progression}

GaCoCo organizes and presents design sessions as missions, with a goal valuable for all. All children should experience a sense of progression through missions, so as to feel more and more competent. To this end, according to their complexity, missions can be chunked into small progressive challenges, disclosed when needed with clear rules, of which the first challenge should be easy to take up by all learners. Progression maps help GaCoCo in tangibly con- 
veying the idea of growth through missions and challenges. An example of a progression map used in the reported study is in Figure 2.

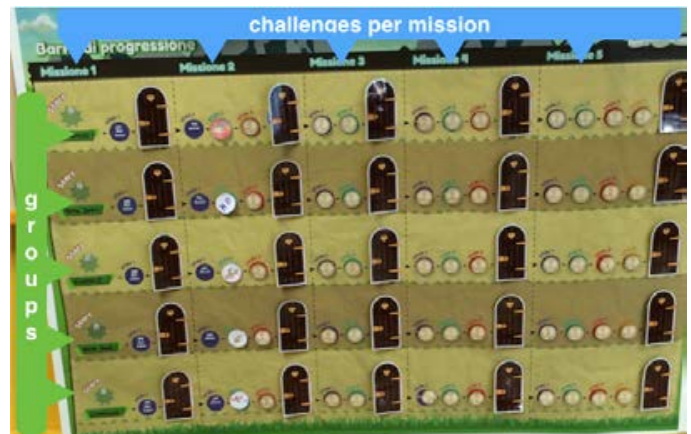

Figure 2. A progression map for each design group (horizontal, green) across design missions with challenges (vertical, blue)

\subsubsection{Feedback System and Control}

Rewards in GaCoCo can be tangible or not and they are part of the so-called feedback system. The gamification literature debates on the benefits of rewards. For instance, in the study reported in (Hanus \& Fox, 2015), rewards contributed to undermining motivations of students interested in the work per se and to increasing competition. Such findings are in line with cognitive evaluation theory, which predicts that a reward can cause people to feel less competent and in control, which decreases intrinsic motivation, when the reward is seen as controlling or not valuable to their work.

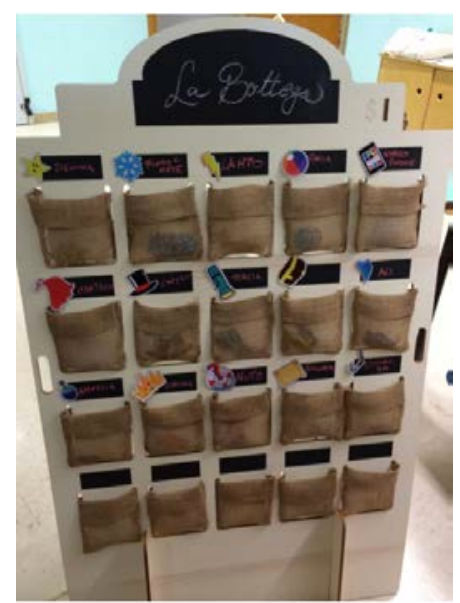

Figure 3. Shop for acquiring design objects

Thus GaCoCo considers only rewards contingent to the design work, so as to be valuable for it, and customized or customizable, achievable on completion of a mission or challenge by all children. Examples of completion-contingent rewards are the objects in the shop in Figure 3. They can be chosen by groups on completion of a mission, independently of who concludes first so as not to increase competition. They serve for prototyping games, so that children perceive that rewards can have a tangible effect on their work. 


\section{Study Design}

\subsection{Aims and Goals}

The aim of the study was to investigate how social relations, measured before and after the game design activity in three different contexts, were linked to positive and negative achievement emotions experienced by children during the activity itself.

\subsection{Hypotheses}

Achievement emotions were measured as referred to a specific context, namely the game design activity conducted at school. This choice is coherent with the assumptions on domain-specificity of achievement emotions within the control-value model (Pekrun, 2006). We evaluated ten discrete achievement emotions, but here, to better explore relationships with social relations, we considered two aggregate indicators, one for positive and one for negative emotions. Social relations were operationalized in terms of two indicators of peer acceptance referred to children's sociometric status. The first indicator is related to the number of choices received by each child; the second indicator is related to the number of reciprocated choices obtained by each child. We hypothesized that both indicators were positively linked to the aggregated indicator of positive emotions and negatively linked to the aggregated indicator of negative emotions.

In addition, we aimed at investigating whether there were differences between the contexts to which the sociometric status referred. Specifically, we asked children to express their social preferences about three contexts differing for their focus on school or leisure activities. We included a school learning context specifically focused on school competence (i.e., class learning activities), an extra-school leisure context specifically focused on free time (i.e., a party), and an intermediate school leisure context (i.e., the school break). Assuming that the game design activities could evoke psychological processes more similar to those involved in leisure rather than school activities, we expected the second context to be more strictly related to the achievement emotions experienced during the game design activity compared to the other two contexts.

\subsection{Participants}

The study involved two classes from two different primary schools in North-Eastern Italy. Children were, in total, 35 (59\% females), coming from a variety of socio-economic backgrounds. Classes were of different ages and sizes: the younger class was of $n=15$ children, in grade 3 , with mean age $=8.85$ years, $\mathrm{SD}=.44$; the older class was of $n=20$ children, in grade 4 , with mean age $=9.72$ years, $\mathrm{SD}=.47$. All children participated on a voluntary basis, and their parents authorized their participation through a written consent form. The study also involved 2 researchers and 2 teachers.

\subsection{Study Protocol}

The study included a game design activity with GaCoCo; pre and post it, specific activities were run and data were gathered, as explained in this section.

\subsubsection{Pre-activity}

During the pre-activity, designers organized a meeting with the school dean and interested teachers in each school in order to explain and discuss the project. A week before the game design activity, a workshop for teachers, lasting cir- 
ca 6 hours, was organized and a more focused training was thus performed. For instance, during the workshop, the expert designer explained the protocol for children, as well as the main ideas of cooperative learning, gamification and game design. Teachers worked in group and experimented the protocol for children by prototyping games themselves. After the workshop, teachers were asked to create heterogeneous groups of children in terms of learning and social skills. Also children were trained to game design principles. This training lasted circa 20 minutes.

At school, teachers administered children a sociometric task, adapted from the literature (Santos, Vaughn, Peceguina, \& Daniel, 2014). This asked each child to choose three classmates, in order of preference, in relation to the three aforementioned contexts, namely a school learning context (e.g., Among your classmates, whom would you like to tackle a task or work with in classroom?), a school leisure context (e.g., Among your classmates, whom would you like to play with during breaks?), and an extra-school leisure context (e.g., Among your classmates, whose birthday party would you like to attend?).

In this manner, for each child and context, before the game design activity, we calculated two indicators of peer acceptance, for a total of six peer acceptance indicators per child (pre). For each context, the first indicator, called received acceptance score, was calculated as the number of first choices received by each child, divided by the number of children included in each class. Similarly, the second indicator, called reciprocated acceptance score, was calculated as the total number of reciprocated choices obtained by each child (i.e., in the cases in which a child was chosen by a peer, who in turn was chosen by the first one), divided by the number of children included in each class. While the first indicator could be thought of as related to social preference, the second could be conceptualized as a mutual friendship indicator.

\subsubsection{Game Design Activity}

The game design activity in each school took a total of five sessions. Each session was organized in a different day of different weeks, and lasted circa two hours and a half. The entire game design activity was organized and conducted in line with GaCoCo (Sect. 2).

Group work was organized with cooperative learning. Strategies for small heterogeneous groups were set in the GaCoCo protocol. Different cooperative learning roles, such as that of ambassador, were assigned by teachers to learners in every design day, according to the protocol, and rotated across missions. Rules for managing group work were explained to the class by their teacher.

Each design day was organized as a gamified mission with a specific goal, using tangible gamified material for conveying a sense of progression, control and relatedness to children. Missions followed a recurring pattern, using the same gamified material. However, each mission challenges had: their own goal, that is, product to release; its specific design material; its cooperative learning strategies.

Specifically, the first mission served to explore rules, roles and create the group badge to use on the progression map across missions, contributing to creating the group identity. The second mission started using cooperative learning strategies for working in pairs and group. It allowed groups to work on their game design idea and specify it in a document, and then, working in pairs, to prototype their characters using a specific frame. The third and fourth missions, working in groups and pairs again, allowed children to work on their game levels, releasing the related document and prototypes. The final mission served to release the document for specifying the progression through game levels, and to assemble levels into a single game prototype per group, thereby presenting it to the class and collecting their feedback.

After each mission, children were administered the Graduated Achievement Emotion Set (GR-AES, Raccanello \& Bianchetti, 2014; Raccanello, Brondino, \& Pasini, 2014). It is a preliminary version of a verbal-pictorial instrument enabling to assess the intensity of ten achievement emotions with children, developed on the basis of Pekrun's con- 
trol-value theory of achievement emotions (Kleine, Goetz, Pekrun, \& Hall, 2005; Lichtenfeld, Pekrun, Stupnisky, Reiss, \& Murayama, 2012; Pekrun, 2006; Pekrun \& Perry, 2014). It allows children to rate an emotion on a 5-point Liker-type scale, with 5 faces corresponding to different levels of emotion intensity ( 1 = not at all and $5=$ extremely). The considered emotions are: three positive activating emotions (enjoyment, hope, pride), two positive deactivating emotions (relief, relaxation), three negative activating emotions (anxiety, anger, shame), and two negative deactivating emotions (boredom, sadness). We calculated the aggregated positive and negative achievement emotions indicators by averaging together the responses related to the five missions for each emotion type (i.e., for the ten achievement emotions) and then summing scores for emotions of the same valence (i.e., for positive and negative emotions).

\subsubsection{Post-activity}

In the post-activity, the same sociometric task of the pre-activity was administered by teachers to children, so as to obtain the six post indicators referred to the three contexts, for both received and reciprocated acceptance scores.

\section{Study Results}

We used SPSS version 20.0 for Windows to calculate descriptive statistics and intercorrelations. We used AMOS version 20.0 to carry out path analyses to test some mediational models. A maximum-likelihood estimation was performed, and a bootstrapping method with a confidence interval was used to test indirect effects. No missing data were present in the dataset.

\subsection{Descriptive Statistics}

We reported the descriptive statistics (means, standard deviations, and intercorrelations) concerning positive and negative achievement emotions and acceptance scores in Table 1 in the Appendix.

\subsection{Achievement Emotions and Sociometric Status}

We conducted four path analyses (separately for positive and negative emotions) to explore the relationships between achievement emotions and received and reciprocated acceptance scores, respectively, as measured pre and post activity. We included pre-acceptance scores in relation to the three contexts (class activities, break, party) as predictors of positive and negative achievement emotions, separately, in turn predictors of the three post-acceptance scores. See Figures 4 and 5 for received scores (top) and reciprocated scores (bottom).

For the four analyses, the direct paths between pre and post-acceptance scores were statistically significant (range of direct paths: from .54 to .81, all ps <.001). For the sake of clarity, these links are not represented in Figures 4 and 5. 

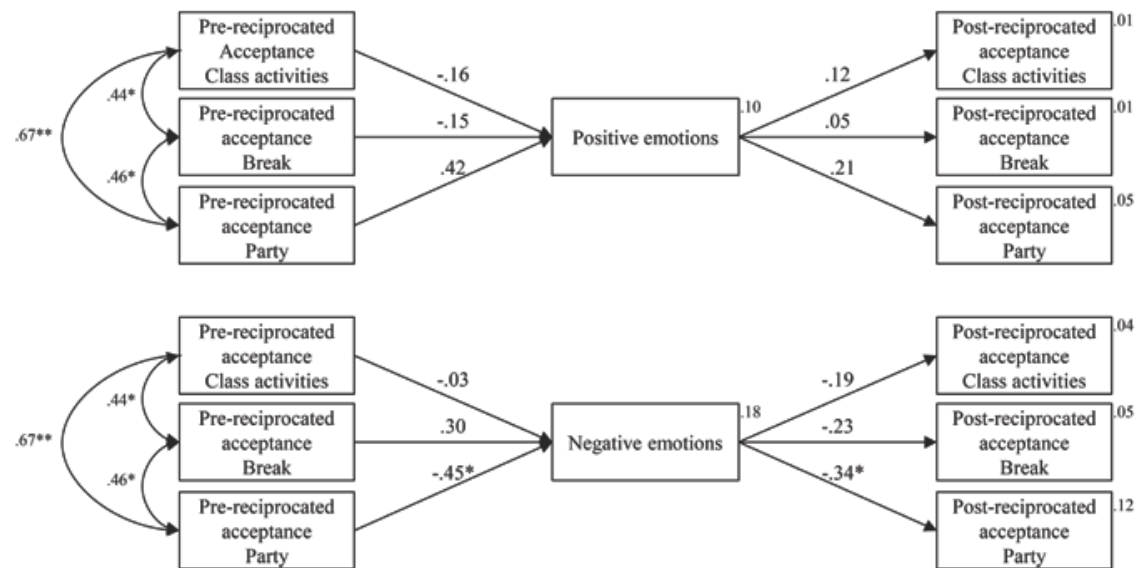

Figure 4. Summary of path analyses for relationships between achievement emotions and pre and postreciprocated acceptance scores, separately for positive and negative emotions, by context (class activities, break, party). Explained variances are reported next to each dependent variable. ${ }^{*} p<.05, * * p<.01, * * * p<$ .001 .
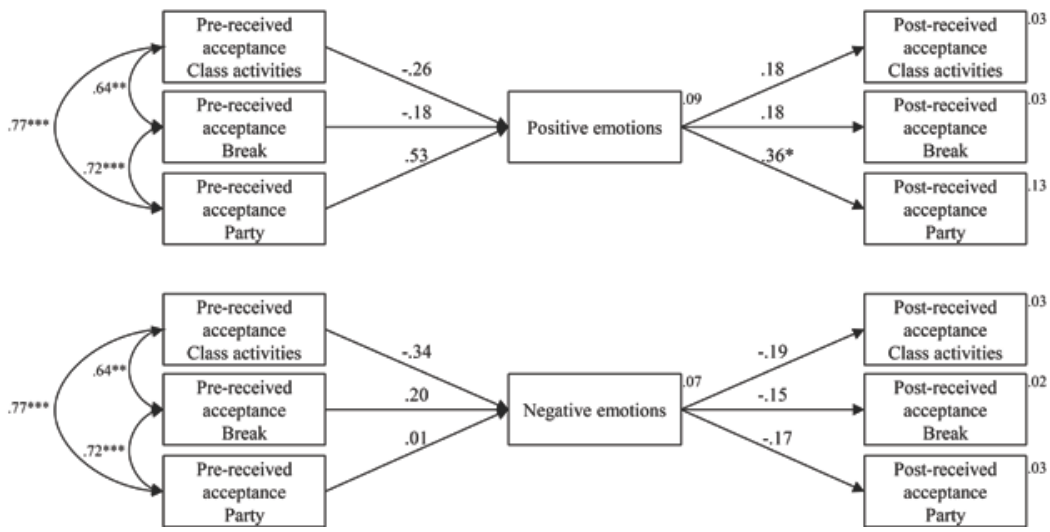

Figure 5. Summary of path analyses for relationships between achievement emotions and pre and postreceived acceptance scores, separately for positive and negative emotions, by context (class activities, break, party). Explained variances are reported next to each dependent variable. ${ }^{*} p<.05$, $* * p<.01, * * * p<.001$.

For received scores, we found that positive emotions positively predicted the post-received acceptance score only for the party context. Even if only this relation resulted statistically significant, it is interesting to note that also postreceived acceptance scores for class activities and break were positively related to positive emotions. In the same way, it is noteworthy that, although for negative emotions no significant paths emerged, these emotions were negatively related to post-received acceptance scores for all three contexts. Finally, no emotion indicator mediated the relationships between pre and post scores, neither totally nor partially.

For reciprocated scores, a different pattern of results emerged. We found that pre-reciprocated acceptance scores negatively predicted negative emotions, which in turn negatively predicted the post-reciprocated acceptance score, again only for the party context. In this case, there was an indirect effect $(.15, p=.13)$, suggesting a possible partial mediating role of negative emotions, even if it was not statistically significant. Although there were only two statistically significant relationships, it is interesting that for all the other cases positive emotions were positively related to postreciprocated acceptance scores, and negative emotions were negatively related, as for received acceptance scores. 


\section{Discussion}

The aim of this work was to investigate the relationships between achievement emotions associated to a game design activity at school and children's peer acceptance before and after the activity. As discussed in the introduction to this paper, currently, from the perspectives of both educational psychology and game design literature, scarce attention has been paid to the links between social competence (Waters \& Sroufe, 1983; Santos et al., 2914) and achievement emotions (Pekrun, 2006; Pekrun \& Perry, 2014), notwithstanding the relevance of such constructs for emotional engagement in group activities, particularly in information technology design contexts.

Our data partially confirmed our hypotheses, according to which we expected positive links between positive emotions and peer acceptance, and vice versa for negative emotions, but significantly for the leisure context concerned with the party. For received choices, positive emotions positively predicted the post-acceptance score. For mutual friendships, the pre-acceptance score negatively predicted negative emotions, which in turn negatively predicted the post-acceptance score. In other terms, negative emotions negatively contributed to the changes in the reciprocated choices, after game design at school, along with a strong role played by the sociometric status previous to the game design activity. On the whole, our findings suggest that positive emotions are more salient for explaining social relations conceptualized in terms of received choices, while negative emotions are more salient when mutual relationships are involved in a design activity with groups of children. In other words, it seems that in order to gain popularity it is sufficient to express positive affect in designing together, but to gain friendships and related privileges children have to be good at regulating their negative affect in designing together.

In addition, it is worth noting that, as expected, the role played by extra-school leisure activities was stronger as compared to the other two activities, suggesting relevant similarities between the way in which children perceive the GaCoCo gamified context and the way with which they represent an usually enjoyable context such as that of a party. This finding is particularly relevant for the design of gamified design activities as in GaCoCo, supporting their emotionally engaging and playful nature.

Finally, the study suffers from limitations related for example to the lack of a control group, to the small sample size, and to the lack of distinctions between discrete emotions and emotions characterizing single missions. Some of these limitations have been addressed in other papers (Brondino et al., 2015; submitted a; submitted b), while others could be tackled in future works including larger sample sizes and control groups.

\section{Conclusions}

This paper briefly presents a game design study in primary schools for realizing paper-based prototypes of games for children, by children, run in Spring 2014. The study used the GaCoCo design method and techniques, organizing design work for groups of children with cooperative learning, and presenting design sessions as missions with specific gamified material. Gamification and cooperative learning were used in the study for soliciting children's emotional engagement and for including all children in the design work. Such constructs (emotional engagement and inclusion) are usually only qualitatively assessed in the design literature with children. The paper, for the first time, presents a way for operationalizing and hence measuring them with quantitative data collection instruments, in the context of the 2014 study, as follows: sociometric status was operationalized considering peer acceptance, as reciprocated and received choices in three different social contexts, whereas emotions were operationalized in terms of achievement emotions.

The paper concludes presenting the results of a series of statistical path analyses for studying the relationships between achievement emotions and peer- acceptance scores. Respectively for indicators of received choices and mutual friendships, positive emotions played a significant role in improving students' sociometric status, while negative emotions were associated with a significant deterioration of the sociometric status, only concerning the leisure context. 
Such data, beyond pointing to the relevance of investigating psychological constructs in specific contexts, support the relevance of fostering positive emotions and reducing negative emotions for favoring inclusion and properly managing group dynamics across a design journey with children.

\section{References}

Brondino, M., Dodero, G., Gennari, R., Melonio, A., Pasini, M., Raccanello, D., and Torello, S., 2015. GaCoCo for emotionally engaging learners at school. In Proceedings of the international conference on Methodologies and Intelligent Systems for Technology Enhanced Learning (mis4TEL'15). Springer.

Brondino, M., Dodero, G., Gennari, R., Melonio, A., Pasini, M., Raccanello, M., and Torello, S., submitted a. "I am happy, I am social, I've realized quality games” or why children's emotions matter in co-design. Journal of Human Computer Studies.

Brondino, M., Dodero, G., Gennari, R., Melonio, A., Pasini, M., Raccanello, M., and Torello, S., submitted b. Engage me, include me, and empower me with game design at school. Computers \& Education.

Cohen, E., 1998. Making cooperative learning equitable. Educational Leadership, 56(1):18-21.

Del Fatto, V., Dodero, G., Gennari, R., Melonio, A., Montali, M., Razniewski, S., Torello, S., Wang, X., and Zini, F., 2014. Gamified children universities: An exploratory study. In Proceedings of the first ACM SIGCHI annual symposium on Computer-human interaction in play (CHI PLAY '14). ACM, New York, NY, USA, 409-410. doi:10.1145/2658537.2661300 http://doi.acm.org/10.1145/2658537.2661300

Dodero, G., Gennari, R., Melonio, A., and Torello, S., 2014a. Gamified co-design with cooperative learning. In CHI '14 Extended Abstracts on Human Factors in Computing Systems (CHI EA '14). ACM, New York, NY, USA, 707-718. doi:10.1145/2559206.2578870 http://doi.acm.org/10.1145/2559206.2578870

Dodero, G., Gennari, R., Melonio, A. and Torello, S., 2014b. Towards tangible gamified co-design at school: Two studies in primary schools. In Proceedings of the first ACM SIGCHI annual symposium on Computer-human interaction in play (CHI PLAY '14). ACM, New York, NY, USA, 77-86. doi:10.1145/2658537.2658688 http://doi.acm.org/10.1145/2658537.2658688

Garzotto, F., 2008. Broadening children's involvement as design partners: from technology to experience. In Proceedings of the 7th international conference on Interaction Design and Children (IDC '08). ACM, New York, NY, USA, 186-193. DOI=10.1145/1463689.1463755 http://doi.acm.org/10.1145/1463689.1463755.

Hanus, M. D., and Fox, J., 2015. Assessing the effects of gamification in the classroom: A longitudinal study on intrinsic motivation, social comparison, satisfaction, effort, and academic performance. Computers \& Education, 80(0):152-161.

Hunicke, R., Leblanc, M., and Zubek, R., 2004. MDA: A formal approach to game design and game research. In Proceedings of Challenges in Games AI Workshop, 19th National Conference of Artificial Intelligence, pages $1-5$.

Kapp, K., 2012. The gamification of learning and instruction. Wiley.

Kagan, M., and Kagan, S., 1992. Cooperative Learning. Kagan Publishing.

Kleine, M., Goetz, T., Pekrun, R. and Hall, N., 2005. The structure of students' emotions experienced during a mathematical achievement test. International Reviews on Mathematical Education, 37:221-225.

Lichtenfeld, S., Pekrun, R., Stupnisky, R. H., Reiss, K., and Murayama, K., 2012. Measuring students’ emotions in the early years: The Achievement Emotions Questionnaire-Elementary School (AEQ-ES). Learning and Individual Differences, 22(2):190-201. doi:10.1016/j.lindif.2011.04.009

Paulus, P., and Nijstad, B., 2003. Group creativity: Innovation through collaboration. Oxford University Press.

Pekrun, R., 2006. The control-value theory of achievement emotions: Assumptions, corollaries, and implications for educational research and practice. Educational Psychology Review, 18(4):315-341. doi:10.1007/s10648-0069029-9. 
Pekrun, R., and Perry, R. P., 2014. Control-value theory of achievement emotions. In R. Pekrun and L. LinnenbrickGarcia (Eds.), International handbook of emotions in education, pages 120-141. New York: Taylor and Francis.

Raccanello, D., and Bianchetti, C., 2014. Pictorial representations of achievement emotions: Preliminary data with primary school children and adults. Advances in Intelligent and Soft Computing, 292:127-134. doi:10.1007/978-3-319-07698-0

Raccanello, D. Brondino, M., and Pasini, M., 2014. Achievement emotions in technology enhanced learning: Development and validation of self-report instruments in the Italian context. Interaction Design and Architecture(s) Journal - IxD\&A, 23:68-81.

Santos, A. J., Vaughn, B. E., Peceguina, I., and Daniel, J. R., 2014. Longitudinal stability of social competence indicators in a Portuguese sample: Q-sort profiles of social competence, measures of social engagement, and peer sociometric acceptance. Developmental Psychology, 50(3):968-978. doi:10.1037/a0034344

Waters, E., and Sroufe, L. A., 1983. Social competence as a developmental construct. Developmental Review, 3(1):79 -97. doi:10.1016/0273-2297(83)90010-2

\section{Appendix}

Summary of correlations, mean values, and standard deviations for positive and negative achievement emotion, prereceived/reciprocated acceptance scores and post-received/reciprocated acceptance scores in the three different contexts (class activities, break, and party).

\begin{tabular}{|c|c|c|c|c|c|c|c|c|c|c|}
\hline Variable & 1 & 2 & 3 & 4 & 5 & 6 & 7 & 8 & $M$ & $S D$ \\
\hline 1. Positive Emotions & -- & -.07 & .05 & -.03 & .24 & .12 & .05 & .21 & -- & -- \\
\hline 2. Negative Emotions & -.07 & -- & -.19 & .08 & -.33 & -.19 & -.23 & $-.34 *$ & -- & -- \\
\hline 3. Pre-acceptance for class activities & .03 & -.20 & -- & $.44^{* *}$ & $.67 * *$ & $.56 * *$ & $.42 *$ & $.56^{* *}$ & .11 & .09 \\
\hline 4. Pre-acceptance for break & .03 & -.01 & $.65 * *$ & -- & $.46^{* *}$ & .25 & $.50 * *$ & $.39 *$ & .10 & .07 \\
\hline 5. Pre-acceptance for party & .19 & -.11 & $.78^{* *}$ & $.72 * *$ & -- & $.66^{* *}$ & $.36^{*}$ & $.67 * *$ & .11 & .06 \\
\hline 6. Post-acceptance for class activities & .18 & -.19 & $.82 * *$ & $.74 * *$ & $.86 * *$ & -- & $.61^{* *}$ & $.59 * *$ & .10 & .07 \\
\hline 7. Post-acceptance for break & .18 & -.15 & $.61^{* *}$ & $.78 * *$ & $.70 * *$ & $.75^{* *}$ & -- & $.48 * *$ & .10 & .07 \\
\hline 8. Post-acceptance for party & $.36 *$ & -.17 & $.61^{* *}$ & $.76^{* *}$ & $.77 * *$ & $.74 * *$ & $.76 * *$ & -- & .09 & .07 \\
\hline$M$ & 13.37 & 9.38 & .22 & .23 & .23 & .22 & .22 & .23 & -- & -- \\
\hline$S D$ & 3.26 & 2.79 & .15 & .15 & .15 & .14 & .15 & .16 & -- & -- \\
\hline
\end{tabular}

Note. Respectively for received and reciprocated acceptance scores, correlations are presented below/above the diagonal; means (standard deviations) in row/column. ${ }^{*} p<.05,{ }^{* *} p<.01$. 